\title{
Colpocleisis: evolución y complicaciones en mujeres del Quindío, Colombia, 2009-2019
}

\section{Colpocleisis: evolution and complications in Quindío women, Colombia, 2009-2019}

\author{
Franklin José Espitia De La Hoz
}

Recibido para publicación: Enero 042020 - Aprobado para publicación: Mayo 062020

\begin{abstract}
RESUMEN
Objetivo. Evaluar y describir los resultados posoperatorios de la colpocleisis en una población de mujeres del Quindío. Materiales y métodos. Estudio de corte transversal. En una población de mujeres mayores de 69 años, con prolapso genital $\geq$ al grado III, sexualmente inactivas y sin interés por preservar la función sexual coital; se les realizó la colpocleisis de LeFort (entre 2009 y 2019). La investigación se efectuó en una institución prestadora de servicios de salud privada, de nivel III, en la ciudad de Armenia. Las mujeres fueron evaluadas antes del procedimiento, luego en las primeras 2 semanas, a los 6 meses, a los 12 meses y después cada año. Resultados. Se operaron 102 mujeres, 69 (67,64\%) por prolapso grado IV y $33(32,35 \%)$ grado III. La edad media fue de 75,39 $\pm 8,46$ años, con un IMC de 27,48 $\pm 7,59$. El tiempo quirúrgico promedio fue 45,93 $\pm 18,63$ minutos. La mediana de la hemorragia transquirúrgica fue de $75 \mathrm{~mL}$. El éxito quirúrgico alcanzado fue del 98,03\%. Conclusiones. La colpocleisis de LeFort, como tratamiento del prolapso genital $\geq$ al grado III, ofrece una tasa de mejoría cercana al 98,03\%, con mínimas complicaciones.
\end{abstract}

Palabras clave: Cuidados posoperatorios, enfermedades de los genitales femeninos, mujeres, prolapso, resultado del Tratamiento.

\begin{abstract}
Objective. To evaluate and describe the postoperative results of colpocleisis in a population of women from Quindío. Materials and methods. Cross-sectional study. In a women population older than 69 years, with genital prolapse $\geq$ grade III, sexually inactive and without interest in preserving coital sexual function; They underwent LeFort colpocleisis (between 2009 and 2019). The research was conducted at a private level III in a health care institution in the city of Armenia. The women were evaluated before the procedure, then in the first 2 weeks, at 6 months, at 12 months, and then every year. Results. 102 women underwent surgery, 69 $(67.64 \%)$ for grade IV prolapse and $33(32.35 \%)$ grade III. The average age was $75.39 \pm 8.46$ years (range $69-96$ ), with a BMI of $27.48 \pm 7.59$ (range $18.36-42.39$ ). The average surgical time was $45.93 \pm 18.63$ minutes. The median transsurgical bleeding was $75 \mathrm{~mL}$. The surgical success achieved was $98.03 \%$. Conclusions. LeFort colpocleisis, as a treatment for genital prolapse $\geq$ grade III, offers an improvement rate of about $98.03 \%$, with minimal complications.
\end{abstract}

Keywords: Postoperative care, genital diseases, female, women, prolapse, treatment outcome.

\section{Citación (Vancouver)}

Espitia De La Hoz FJ. Colpocleisis: evolución y complicaciones en mujeres del Quindío, Colombia, 2009-2019. Rev Avances en Salud; 2020. (4)1:12-23. doi: 10.21897/25394622.1924
() 2020. Universidad de Cordoba. Este es un artículo de acceso abierto distribuido bajo los términos de la licencia Creative Commons Attribution License, que permite el uso ilimitado, distribución y reproducción en cualquier medio, siempre que el autor original y la fuente se acreditan. 


\section{INTRODUCCIÓN}

El prolapso de los órganos pélvicos (POP) es definido como la protrusión de los órganos pélvicos (vejiga, útero, cúpula vaginal y/o recto) en el canal vaginal o hacia fuera de este (1). Es una condición de prevalencia in crescendo a medida que aumenta la edad. Se presume que alrededor del $6,4 \%$ de las mujeres de 60 o más años, se someterán a una cirugía reconstructiva del piso pélvico a consecuencia del POP, de las cuales $1 / 3$ tiene riesgo de ser sometidas a una segunda cirugía para el tratamiento de la recurrencia (2). En Colombia, la prevalencia del POP se ha reportado en el $31,62 \%$ de las mujeres no histerectomizadas y en el $68,38 \%$ de las histerectomizadas, en donde el POP grado III se ha estimado en $14,3 \%$, mientras que para el grado IV es del 3,37 \% (1).

El diagnóstico del POP es clínico, orientando la evaluación de acuerdo a la sintomatología referida por la mujer, caracterizada principalmente por la sensación y/o percepción de cuerpo extraño o bulto vaginal, lo cual le puede o no generar discomfort, pudiendo acompañarse de síntomas urinarios y/o de la defecación $(1,3)$. El abordaje terapéutico del POP es de carácter individual, y dependerá tanto de lo incapacitante de la sintomatología como del impacto en la calidad de vida de cada mujer, así como de la edad, condiciones clínicas y perspectivas de su vida sexual en el futuro. El tratamiento cuenta con alternativas conservadoras (rehabilitación del piso pélvico) y el uso de pesarios, sin ser una solución definitiva del defecto; sin embargo, el manejo del POP sintomático es principalmente quirúrgico, pues la cirugía es la indicación ante severos defectos anatómicos y/o funcionales $(1,3,4)$.

Las técnicas quirúrgicas se reúnen en 2 grupos: a) Cirugía reconstructiva (corrige el POP, restableciendo la anatomía; ya sea utilizando los propios tejidos de la mujer, -con o sin el concurso de estructuras autólogas o material protésico-); b) Cirugía obliterativa (corrige el POP a través de la cancelación y/o cierre parcial o total de la vagina) (5-7).

La colpocleisis, es la técnica quirúrgica que caracteriza a la cirugía obliterativa vaginal; fue descrita y modificada por LeFort en 1877 (8), desde entonces se ha convertido en una excelente alternativa en mujeres, sexualmente inactivas, en donde preservar la función sexual vaginal no es prioridad, y que se caracterizan por tener altos riesgos quirúrgicos (9). Las tasas de recurrencias son poco comunes $(0-7 \%)$, mientras la prevalencia de incontinencia urinaria de urgencia o de esfuerzo de novo registra porcentajes variables (0 a $26 \%$ y 0 a $10 \%$, respectivamente) (10).

La colpocleisis ofrece como ventajas: bajo riesgo de morbilidad perioperatoria, técnica rápida y fácil de realizar con disminución del tiempo quirúrgico, menor pérdida sanguínea, rápida recuperación posoperatoria, bajo riesgo de recurrencia del prolapso, bajas tasas de arrepentimiento, los canales transversales y laterales (facilitan el drenaje de las secreciones), y se puede practicar con anestesia local, regional o general superficial; además de ser respetablemente aceptada por la mayoría de los ginecólogos para el tratamiento de prolapsos severos en mujeres de edad avanzada (sin vida sexual coital), y que por sus comorbilidades médicas no son candidatas para otras opciones terapéuticas. Se destacan como desventajas: impide la actividad sexual coital vía vaginal, se asocia con altas tasas de incontinencia urinaria de esfuerzo "de novo" e impide la accesibilidad vaginal del cérvix y del útero (11-13), pudiendo retrasar el diagnóstico de una neoplasia maligna ginecológica.

El propósito de la presente investigación consiste en evaluar los patrones de la práctica actual en una institución privada -de tercer 
nivel de complejidad- de Armenia y describir los resultados posoperatorios de la colpocleisis en una población de mujeres del Quindío.

\section{MATERIALES Y MÉTODOS}

Estudio de corte transversal, en mujeres a las cuales se les realizó la colpocleisis de LeFort, entre el 01 de febrero del 2009 y el 28 de febrero del 2019. El estudio fue realizado en una clínica privada de Armenia, -capital del departamento del Quindío (ubicado en la región central de Colombia)-, centro de referencia, de carácter universitario, que atiende a personas pertenecientes a los regímenes de aseguramiento contributivo y subsidiado por el Estado, en el sistema de seguridad social. Se incluyó una población de 102 mujeres. Se hizo muestreo consecutivo, intentando la inclusión de todas las intervenidas en el periodo de estudio.

Procedimiento: Las mujeres fueron operadas por un especialista entrenado en Ginecología Urológica y Reconstrucción del Piso Pélvico. Se incluyeron mujeres mayores de 69 años, con prolapso genital $\geq$ al grado III, sexualmente inactivas y sin interés por preservar la función sexual coital. Se excluyeron mujeres que no contaron con toda la información en la historia clínica y seguimiento menor a 6 meses. Se identificaron por el código de diagnóstico CIE-10 N813 a partir de una base de datos. El prolapso genital se clasificó, en el preoperatorio, mediante el sistema de estadificación del prolapso de los órganos pélvicos conocido como POP-Q (Pelvic Organ Prolapse Quantification System) (14). Para medir la satisfacción de la paciente después de la reparación quirúrgica, se utilizó la Escala de Impresión de Mejoría Global del Paciente (PGI-I); el cual es un cuestionario de autoevaluación, donde la simplicidad y la brevedad son su principal característica; está dirigido específicamente a la evaluación de la satisfacción del paciente después de las terapias urológicas, incluido el tratamiento de los síntomas del tracto urinario inferior, la reparación quirúrgica de la incontinencia urinaria de esfuerzo, el prolapso urogenital, etc. El PGI-I evalúa en una sola pregunta (solicita clasificar el alivio obtenido con el tratamiento), según una escala de Likert de siete puntos ( 1 a 7 , donde 1 representa "Muchísimo mejor" y 7 "Muchísimo peor"). El tratamiento es considerado "con éxito", si responden "Muchísimo mejor" o "Mucho mejor"; las otras opciones de respuesta se definen como "fallo de tratamiento". $(15,16)$.

Técnica quirúrgica: A cada participante se le practicó cerclaje anal (al inicio de la cirugía, con sutura de seda 0 para asegurar un campo operatorio lo más limpio posible). La evacuación vesical se realizó con sonda Foley. La profilaxis antibiótica se hizo con amoxicilina-clavulánico, un gramo media hora antes de la cirugía. En el caso de alergia a la penicilina se utilizó clindamicina 1,2 gramos. La ubicación de la paciente se realizaba en posición de litotomía (avanzando la región glútea ligeramente por fuera del borde de la cama quirúrgica), de tal manera que todo el grupo quirúrgico se ubique en un mismo plano, al frente del periné.

La analgesia administrada consistió en 1 gramo de dipirona cada 6 horas y $50 \mathrm{mg}$ de tramadol por vía subcutáneo cada 8 horas, únicamente en el primer día. En el segundo día se usó analgesia oral con acetaminofén ( 1 gramo por vía oral cada 8 horas).

Se utilizó la técnica de LeFort modificada (8). Se conserva el cuello y cuerpo uterino (en las mujeres con útero, ya que está demostrado que no existe ventaja alguna en retirar el útero, a expensas de prolongar el tiempo quirúrgico), se les dejaban túneles de epitelio a ambos lados de la vagina, para facilitar la visualización del flujo genital.

Se describen 6 pasos progresivos: 1) con el cuello pinzado y en tracción sostenida, se calculan dos rectángulos de mucosa vaginal a resecar de $6-15 \mathrm{cms}$ de largo por $5-7$ cms de ancho, uno anterior y otro posterior, comenzando a $1-2 \mathrm{cms}$ por encima del orificio externo del cérvix y llegando hasta un 
$1-2 \mathrm{cms}$ del meato uretral, exponiendo la fascia vesico-vaginal; y $2-3 \mathrm{cms}$ del reborde muco-cutáneo de la horquilla recto-vaginal subyacente; dejando un espacio lateral de 2 - 3 cms cubierto con mucosa, a cada lado, a fin de permitir túneles de drenaje epitelizados, además de mantener libres $2-3 \mathrm{cms}$ en la pared anterior, correspondientes a la uretra y su previsible unión uretro-vesical; 2) se fija una serie de 3 - 4 puntos de sutura poliglactina 910, sagitalmente desde la fascia vesico-vaginal hacia la fascia recto-vaginal para aproximar las porciones mediales de los rectángulos, tratando de invaginar las fascias; 3 ) el cierre se continúa creando canales laterales suturando la fascias hasta el límite del borde epitelial a cada lado, hasta la invaginación total del prolapso; 4) se realiza el afrontamiento de los bordes de mucosa vaginal anterior y posterior, con lo cual se reduce el prolapso, sellando de esta forma la vagina; 5) se procede a la realización de cirugía anti-incontinencia según la necesidad; 6) se practica una colpoperineorrafia posterior a fin de disminuir el hiato genital y reforzar el cuerpo y membrana perineal, además de mejorar el aspecto estético. En todos los casos, la cirugía fue realizada por el mismo especialista, asistido por un ayudante quirúrgico entrenado en la técnica.

Las mujeres fueron evaluadas antes del procedimiento, luego en las primeras 2 semanas, a los 6 meses, a los 12 meses y después cada año. En cada control se les solicita a las participantes diligenciar la Escala de Impresión de Mejoría Global del Paciente (PGI-I).

Variables medidas: socio-demográficas, antecedentes gineco-obstétricos y antecedentes quirúrgicos, síntomas asociados antes de la cirugía y las comorbilidades. También se determinaron las características del procedimiento (tiempo quirúrgico y sangrado), los hallazgos quirúrgicos, los procedimientos realizados, la estancia hospitalaria, la presencia de complicaciones quirúrgicas y la Escala de Impresión de Mejoría Global del Paciente (PGI-I).
Análisis estadístico: Se creó una base de datos en Excel con un formulario de recolección de datos previamente diseñado, validado por tres expertos clínicos. Se utilizó estadística descriptiva. Para las variables cuantitativas se calculó la mediana, rangos, media y la desviación estándar (DE) y para las cualitativas se utilizaron tablas de frecuencias. Para el procesamiento de datos se utilizó SPSS versión 14.0

Aspectos éticos: Este estudio fue aprobado por el comité de ética de Hathor, Clínica Sexológica (Acta No. 03). Se siguieron los principios éticos de la declaración de Helsinki y la Resolución 8430 de 1993 del ministerio de Salud de Colombia (Artículos 5 y 11). Se garantizó la confidencialidad y privacidad de la información.

\section{RESULTADOS}

Siguiendo los datos en la oficina de Estadística de la Clínica donde se realizó el estudio, entre el 01 de febrero de 2009 y el 28 de febrero de 2019 se presentaron 21.807 atenciones con diagnóstico de patología benigna ginecológica. De estas, $5.928(27,18 \%)$ tenían diagnóstico de prolapso de los órganos pélvicos (POP) (Código CIE-10 N811 - N818), -3.906 (65,89\%) tenían antecedente de histerectomía (65,82 \% vaginal, $33,48 \%$ abdominal y 0,69 \% laparoscópica-). De las 5.928 mujeres con POP, 4.167 (70,29 \%) fueron Ilevadas a cirugía, de las cuales 123 $(2,95 \%)$ se le realizó la colpocleisis; 1149 $(19,38 \%)$ recibieron manejo conservador (rehabilitación del piso pélvico) y 612 (10,32 $\%)$ se trataron con el uso de pesarios vaginales de silicona.

De las 123 mujeres a las que se les realizó la colpocleisis, se excluyeron 9 por no cumplir los criterios inclusión, 7 se mudaron de ciudad, 3 no desearon participar y 2 no fue posible contactarlas; al final quedaron 102 mujeres para hacer parte del estudio. La media del tiempo de seguimiento fue de 34,29 $\pm 13,56$ 
meses.

La edad media fue de 75,39 \pm 8,46 años, mientras que la edad promedio de la pareja fue de 79,86 $\pm 8,79$ años. El promedio de la edad de la menopausia fue de 47,96 $\pm 8,19$ años. El IMC fue de 27,48 $\pm 7,59$. El 42,19 \% tenían pareja estable, el 65,68\% residían en área rural y $87,25 \%$ pertenecían al régimen contributivo. El 36,27 \% tenían antecedente de cirugía reconstructiva del piso pélvico. En la tabla 1 se muestran los datos socio-demográficos.

Tabla 1. Características socio-demográficas de las mujeres sometidas a colpocleisis en el Quindío, Colombia, 2009-2019.

Variable y categorías

\begin{tabular}{lc}
\hline Edad: $X \pm$ DE años & $75,39 \pm 8,46$ \\
Peso: $X \pm$ DE Kg & $68,43 \pm 8,73$ \\
Talla: $X \pm$ DE Cms & $156,93 \pm 8,16$ \\
IMC: $X \pm$ DE & $27,48 \pm 7,59$ \\
Raza
\end{tabular}

Hispánicas

$58(56,86 \%)$

Indígenas

$7(6,86 \%)$

Afrocolombianas $\quad 37(36,27 \%)$

Estrato socio-económico

\begin{tabular}{lc} 
Alto & $9(8,82 \%)$ \\
\hline Medio & $49(48,03 \%)$ \\
\hline Bajo & $44(43,13 \%)$
\end{tabular}

Ocupación

\begin{tabular}{lc} 
Amas de casa & $96(94,11 \%)$ \\
\hline Empleadas & $6(5,88 \%)$
\end{tabular}

Nivel de estudios

Primaria

$32(31,37 \%)$

Elaboración propia. 


\begin{tabular}{ll}
\multicolumn{1}{c}{ Variable y categorías } & Promedio \\
\hline Secundaria & $24(23,52 \%)$ \\
\hline Técnicos & $29(28,43 \%)$ \\
\hline Profesionales & $17(16,66 \%)$ \\
\hline Hábitos & \\
\hline Ingesta de alcohol & $79(77,45 \%)$ \\
\hline Tabaquismo & \\
\hline Fumadora, n (\%) & $23(22,54 \%)$ \\
\hline Exfumadoras, $\mathrm{n} \mathrm{( \% )}$ & $28(27,45 \%)$ \\
\hline Sedentarismo & $83(81,37 \%)$ \\
\hline
\end{tabular}

Elaboración propia.

Con relación a los antecedentes médicos el 100 $\%$ presentaban comorbilidades médicas. En la tabla 2 se presenta la distribución de las enfermedades crónicas agrupadas según CIE-10 con frecuencia porcentual. El 61,63\% (n=63) sufría 2 o más comorbilidades médicas. El 10,78 \% no refirió ningún antecedente quirúrgico; 7,84 \% tenía cirugía por embarazo ectópico; 36,27 $\%$ bloqueo tubárico bilateral, 12,74 \% salpingectomía unilateral y 9,8\% salpingectomía bilateral.

En cuanto al riesgo anestésico, 75,49 \% fueron

Tabla 2. Enfermedades crónicas en mujeres sometidas a colpocleisis en el Quindío, Colombia, 2009-2019.

\begin{tabular}{ll}
\multicolumn{1}{c}{ Patología } & Prevalencia \\
\hline HTA & $72,54 \%$ \\
\hline Dislipidemia & $65,68 \%$ \\
\hline Enfermedad cardiovascular & $27,45 \%$ \\
\hline Diabetes & $18,62 \%$ \\
\hline Osteoporosis & $13,72 \%$ \\
\hline $\begin{array}{l}\text { Infecciones de la vía urinaria a } \\
\text { repetición }\end{array}$ & $12,74 \%$ \\
\hline Hipotiroidismo & $10,78 \%$ \\
\hline Neumopatías crónicas & $8,82 \%$ \\
\hline Enfermedad de Parkinson & $1,96 \%$ \\
\hline Enfermedad de Alzheimer & $0,98 \%$ \\
\hline
\end{tabular}

Elaboración propia. 
clasificadas como ASA III / IV. El tratamiento hormonal local se prescribió en el 88,23 \%.

El tiempo de evolución con el prolapso de las participantes arrojó una media de 7,29 \pm 4,83 años. Los tres síntomas que se detectaron con mayor frecuencia fueron: masa genital palpable (100\%), disfunción defecatoria (constipación o digitación) $(72,54 \%)$ y obstrucción urinaria (70,58 \%). El promedio de tiempo sin actividad coital fue de 11,37 $\pm 5,43$ años.

El 68,62\% había tenido más de nueve embarazos, reportándose en el total de la población, una mediana de 9 embarazos (rango entre 0 - 21). La paridad reportó una mediana de 6 (rango 0 - 15), con una mediana de 6 partos vaginales (rango 0 - 15), y una mediana de 2 cesáreas (rango 0 - 9). El parto instrumentado se encontró en el 5,88 \% del total de los partos vaginales.

De las 102 mujeres participantes, 69 (67,64\%) presentaban prolapso grado IV y 33 (32,35\%) grado III. El 41,17\% (n=42) habían sido sometidas a histerectomía, 85,71\% $(n=36)$ vaginal y
14,28\% (n=6) abdominal. El 18,62 \% habían sido sometidas a uretropexia tipo Burch, el $38,23 \%(n=39)$ colporrafia anterior y posterior.

Al 76,47 \% ( $n=75)$ se le encontró un prolapso anterior y al 51,96 \% ( $n=53)$ un prolapso posterior; y todas tenían prolapso apical en etapa III o IV. La prevalencia de desgarro perineal fue del 58,82\%, más frecuente en las mujeres con 2 o más partos vaginales, así como en aquellas cuyo parto fue atendido en casa. El prolapso rectal se hizo presente en el 6,86\% $(n=7)$. Las mediciones preoperatorias del POPQ, se reportan en centímetros, y se describen en la tabla 3.

El 38,23\% $(n=39)$ presentaban incontinencia de esfuerzo, $17,64 \%(n=18)$ incontinencia de urgencia y $26,47 \%(n=27)$ incontinencia urinaria oculta. El 32,14\% $(n=27)$ de las incontinencias diagnosticadas con estudio de urodinamia, fueron llevadas a corrección quirúrgica. Al 55,55\% $(n=15)$ se les corrigió con sling TVT (Tension-free vaginal tape) y al 44,44\% $(n=12)$ con el sling TOT (Transobturator tape).

Tabla 3. Enfermedades crónicas en mujeres sometidas a colpocleisis en el Quindío, Colombia, 2009-2019.

\begin{tabular}{|c|c|}
\hline POP-Q & Medidas \\
\hline Aa & $+2,79 \pm 1,11$ \\
\hline $\mathrm{Ba}$ & $+5,16 \pm 1,53$ \\
\hline C & $+8,49 \pm 2,79$ \\
\hline gh & $4,89 \pm 0,96$ \\
\hline $\mathrm{pb}$ & $2,76 \pm 1,23$ \\
\hline Tvl & $9,36 \pm 4,29$ \\
\hline Ap & $+2,46 \pm 0,84$ \\
\hline $\mathrm{Bp}$ & $+4,29 \pm 0,69$ \\
\hline $\mathrm{D}$ & $+5,19 \pm 1,83$ \\
\hline
\end{tabular}

Elaboración propia. 
A ninguna de las mujeres se les realizó cistoscopia prequirúrgica y el estudio de urodinamia solo se ordenó en el 55,88 \% $(n=57)$ del total de las participantes.

El procedimiento quirúrgico se hizo con anestesia regional en el $100 \%$ de los casos. El tiempo quirúrgico promedio fue 45,93 $\pm 18,63$ minutos. La mediana de la hemorragia transquirúrgica fue de $75 \mathrm{~mL}$ (rango 50 - $150 \mathrm{~mL}$ ).

La estancia hospitalaria posquirúrgica en promedio alcanzó una mediana de 1 día (rango 1 - 3). El 88,23\% de las participantes se les dio de alta antes del tercer día del postoperatorio.

El $8,82 \%(n=9)$ presentaron complicaciones (3 infección urinaria baja - fueron tratadas con antibióticos, con total recuperación-; 2 retención urinaria - cedió con el uso de sonda vesical por siete días, con vaciamiento programado cada tres horas-; 2 hematomas de cúpula -tratado de manera expectante-; 2 recidivas del prolapso -requirió otro manejo quirúrgico-, el tiempo promedio desde la operación hasta la recurrencia fue de 6,39 meses). No hubo complicaciones relacionadas con la TOT o TVT en las mujeres en las que se utilizó esta técnica, ni tampoco hubo complicaciones relacionadas con la anestesia.

No hizo falta administrar analgesia adicional a la prescripción inicial, a fin de controlar el dolor postoperatorio.

La frecuencia de incontinencia urinaria, en el postoperatoria, fue de $21,56 \%(n=22)$ y 12,74 $\%(n=13)$ para la de urgencia y de esfuerzo "de novo", respectivamente. A los 12 meses de seguimiento, se observó entre las mujeres operadas por incontinencia urinaria de esfuerzo, una tasa de recurrencia postquirúrgica del 15,68\%.

En ningún caso se detectó arrepentimiento por la pérdida de la función sexual coital, no obstante, se observó que el 10,78 \% volvió a la práctica de la sexualidad sin penetración.

A los 12 meses de seguimiento, según la Escala de Impresión de Mejoría Global del Paciente (PGI-I), el 94,11 \% ( $n=96)$ manifestaron estar "muchísimo mejor", el 3,92 \% (n=4) "mucho mejor" y el 1,96 \% (n=2) "ningún cambio". Registrándose un éxito quirúrgico del 98,03 \%, con un nivel de satisfacción del 94,11\% con los resultados postoperatorios (funcionales y anatómicos).

\section{DISCUSIÓN}

En esta investigación se describe la evolución y los desenlaces del tratamiento del prolapso de los órganos pélvicos con la técnica obliterativa (colpocleisis de LeFort) en 102 mujeres del Quindío, en un periodo de 10 años. Las cuales corresponden al 2,95\% del total de las pacientes que fueron sometidas a cirugía del piso pélvico en el periodo de seguimiento.

En el presente estudio se identificó que el 27,18 $\%$ de las mujeres consultantes del servicio de ginecología, tenían diagnóstico de prolapso de los órganos pélvicos (POP), encontrándose en el rango del 31,8 \% reportado por Handa et al. (17) en E.E.U.U. en una cohorte de 412 participantes, pero superior al 23,19\% descrito por Espitia et at. (1) en una población del Eje Cafetero (Colombia).

La edad media de este grupo fue de 75,39 \pm 8,46 años, similar a lo reportado en otras poblaciones de mujeres sometidas a colpocleisis de LeFort, en países como Colombia, Chile y Portugal (18-20).

Los antecedentes médicos se hicieron presentes en el $100 \%$ de las participantes, donde el $61,63 \%$ (presentó 2 o más comorbilidades); cercano al 97,9\% descrito por Hernández et al. (18) en Medellín (Colombia), aunque inferior al 69,8\% mencionado por Marín et al. (13) en Bogotá (Colombia).

Los síntomas más frecuentes caracterizaron la masa genital palpable, disfunción defecatoria y obstrucción urinaria, lo cual con cuerda con lo publicado por Espitia et al. $(1,3,21)$, en el Eje Cafetero (Colombia); resultado ya descrito por otros autores $(13,18)$.

El tiempo de evolución del prolapso arrojó una media de 7,29 \pm 4,83 años, menor a lo publicado en Colombia por Marín et al. (13), en un grupo de 53 mujeres. Este autor informa de mujeres cuyo prolapso estuvo presente hasta por 
40 años, al momento de la cirugía, afirmando la influencia cultural a la hora de buscar ayuda por conflictos relacionados con los genitales. Es importante mencionar que al 5,88 \% del total de las participantes se les atendió el parto instrumentado, siendo este porcentaje superior al 1,17\% descrito por Espitia et al. (22) en Armenia (Colombia), en una población de 27 mujeres con procidencia genital completa.

En nuestra serie, la mayoría de las mujeres $(67,64 \%)$ presentaban prolapso grado IV, similar a lo reportado por Zebede et al. (23) en una serie de 325 mujeres sometidas a colpocleisis LeFort, en Cleveland Clinic Florida. A su vez el prolapso rectal se hizo presente en el 6,86 $\%$ de las participantes, muy superior a lo descrito por Hatch et al. (24) en E.E.U.U. el cual describe que el prolapso rectal se produce con poca frecuencia, con una incidencia en adultos de $0,25-0,42 \%$ y una prevalencia de $1 \%$ en adultos mayores de 65 años de edad. En nuestra serie lo asociamos a la diástasis y debilidad de la musculatura del suelo pelviano y del canal anal, característico en este grupo poblacional. El 98,03 \% de éxito quirúrgico alcanzado en este estudio, se encuentra dentro del rango reportado en la literatura $(12,13,18)$. Acerca de las recidivas, el 1,96\% descrito en nuestro estudio, es inferior al 4,2\% informado por Mikos et al. (25) en una revisión publicada en el 2016, donde se incluyeron 28 estudios y 1.810 pacientes; ratificándola como una técnica quirúrgica representativa dentro del arsenal a utilizar, en mujeres mayores con prolapso avanzado, que no son candidatas para otros procedimientos reconstructivos.

Las complicaciones en esta investigación son del $8,82 \%$, similar al 8,1\% informado por Catanzarite et al. (26) en 283 mujeres de Chicago, Illinois (E.E.U.U.), pero inferior al 15,2\% descrito por Zebede et al. (23), aunque en este último estudio se trató de un grupo de mujeres cuya edad media fue de 81,3 \pm 5,3 años.

En otras publicaciones $(18,23,27)$ la realización de cirugía anti-incontinencia muestra porcentajes superiores al 32,14\% reportado por nosotros. La diferencia se puede explicar porque, en nuestra investigación, solo se operaron las mujeres a las cuales se les había diagnosticado la incontinencia urinaria por urodinamia. En cuanto al tiempo quirúrgico promedio, nuestros resultados son menores a los presentados por Krissi et al. (28) en Tel Aviv (Israel), que reportan una duración media de 59,6 9, 1 minutos, y mucho menor que a los 121,5 \pm 31,9 minutos reportados por Park et al. (29) en Seúl (Korea), en una cohorte de 95 mujeres; lo cual se explica por la simplicidad de la técnica quirúrgica aplicada en esta investigación.

El tipo de anestesia utilizado en este estudio fue regional (100\% de los casos), resultado superior a lo informado por otros autores $(13,18,23,26)$; lo que se entiende por el tipo de población intervenida por cada autor, en el afán de buscar la prevención de la morbilidad y mortalidad postoperatoria, en una población de mujeres severamente enfermas y de edad tan avanzada. El 94,11\% del nivel de satisfacción encontrado en nuestro estudio, es similar a las representativas tasas de satisfacción reportadas por otros autores $(12,18,26,29)$, posicionado la colpocleisis como la cirugía de elección, en mujeres de edad avanzada que tienen múltiples comorbilidades.

La estancia hospitalaria posquirúrgica arrojó una mediana de 1 día, menos a lo reportado por Hernández et al. (18), o a los 3,5 $\pm 1,5$ días descritos por Krissi et al. (28); esto permite optimizar los recursos sanitarios, disminuir la morbimortalidad, el deterioro funcional y la institucionalización derivada de procedimientos quirúrgicos mayores, lo cual posiciona el procedimiento de cirugía obliterativa, como la menos invasiva y cuyos resultados son más duraderos, entre todas las cirugías reconstructivas del piso pélvico disponibles (30).

Finalmente, no recomendamos la histerectomía concurrente, al mismo tiempo de la colpocleisis, por las siguientes razones: 1) potencia el riesgo de complicaciones; 2) incrementa el sangrado intraoperatorio; 3) prolonga el tiempo quirúrgico. En lo cual existe concordancia con otros autores $(12,31,32)$.

El significativo $10,78 \%$ de práctica sexual sin penetración, en el posoperatorio, es explicable porque, en las mujeres de nuestra consulta, se promueve y empodera la importancia de la salud sexual en el adulto mayor, lejos de la geni- 
talidad, tal como lo hemos publicado (33). La principal limitación de esta investigación consiste en no haber tenido un grupo control para comparar, por lo tanto, los resultados y observaciones deberán ser confirmadas con la realización de un ensayo clínico controlado donde se compare la técnica de la colpocleisis (aquí realizada) con alguna de las otras técnicas descritas en la literatura médica. La más significativa fortaleza radica en el importante número de mujeres intervenidas, además la técnica quirúrgica empleada en el estudio, permite ser aplicada y generalizarla en cualquier tipo de mujer con prolapso genital del grado III /IV.

\section{CONCLUSIONES}

La colpocleisis de LeFort, es una técnica quirúrgica efectiva en el tratamiento del prolapso de órganos pélvicos $\geq$ al grado III; sin la necesidad de instrumental quirúrgico complejo, con bajas complicaciones; ofreciendo una tasa importante de mejoría y satisfacción a lo largo del tiempo; lo que la hace una alternativa segura a considerarse como la mejor opción de tratamiento en el prolapso genital severo en mujeres de edad avanzada, sin interés por la actividad sexual coital.

\section{CONFLICTO DE INTERÉS}

Los autores declaran no tener conflicto de interés y no haber recibido ningún tipo de patrocinio para llevar a cabo esta investigación.

\section{FINANCIACIÓN}

La investigación fue diseñada y financiado por el autor principal, el cual realizó todas las cirugías.

\section{AGRADECIMIENTOS}

Le ofrecemos especial agradecimiento a Hathor, Clínica Sexológica, por facilitarnos llevar a cabo esta investigación; al Dr. Carlos Díaz Támara (maestro y amigo), por darnos las herramientas para lograr el nivel de instrucción quirúrgica que hemos alcanzado, y por compartir con nosotros el espíritu investigativo que lo caracteriza. Al doctor Álvaro Enrique Ochoa
Cuberos (ferviente defensor de la cirugía vaginal), por sus valiosos aportes en la revisión de este manuscrito.

\section{REFERENCIAS}

1. Espitia-De La Hoz FJ. Factores de riesgo asociados con prolapso genital femenino: estudio de casos y controles. Urol Colomb. 2015; 24(1):12-8. doi: https://doi.org/10.1016/j.uroco.2015.03.003

2. Olsen AL, Smith VJ, Bergstrom JO, CoIling JC, Clark AL. Epidemiology of surgically managed pelvic organ prolapse and urinary incontinence. Obstet Gynecol. 1997; 89(4):501-6. doi: 10.1016/S00297844(97)00058-6

3. Espitia-De La Hoz FJ. Complicaciones del uso del pesario con soporte y sin soporte en el prolapso genital completo. MÉD UIS. 2015; 28(3):309-15.doi: http://dx.doi. org/10.18273/revmed.v28n3-2015005

4. Wagenlehner FM, Bschleipfer T, Liedl B, Gunnemann A, Petros P, Weidner W. Surgical reconstruction of pelvic floor descent: anatomic and functional aspects. Urol Int. 2010; 84(1):1-9. doi: 10.1159/000273458

5. Barber MD, Brubaker L, Nygaard I, Wheeler TL, Schaffer J, Chen Z, et al. Defining success after surgery for pelvic organ prolapse. Obstet Gynecol. 2009; 114(3):600-9. doi: 10.1097/AOG.0b013e3181b2b1ae

6. Bjelic-Radisic V, Aigmueller T, Preyer $\mathrm{O}$, Ralph G, Geiss I, Müller G, et al. Vaginal prolapse surgery with transvaginal mesh: results of the Austrian registry. Int Urogynecol J. 2014; 25(8):1047-52. doi: https://doi. org/10.1007/s00192-014-2333-x

7. Vij M, Bombieri L, Dua A, Freeman Rology Working Group. Long-term follow-up after colpocleisis: regret, bowel, and bladder function. Int Urogynecol J. 2014; 25(6):8115. doi: https://doi.org/10.1007/s00192-0132296-3

8. LeFort L. Nouveau procede pour la guerison du prolapsus uterin. Bull Gene Ther. 1877; 92:337-46. Recuperado a partir de: https://ci.nii.ac.jp/naid/10010110468/en/

9. Harmanli $\mathrm{OH}$, Dandolu $\mathrm{V}$, Chatwani AJ, 
Grody MT. Total colpocleisis for severe pelvic organ prolapse. J Reprod Med. 2003 Sep;48(9):703-6. Recuperado a partir de: https://europepmc.org/article/med/14562635

10. Deffieuxa X, Thubertb T, Dononc L, Hermieud JF, Leormande L, Trichotf C. Chirurgie d'occlusion vaginale (colpocléisis) pour prolapsus génital: recommandations pour la pratique clinique Colpocleisis: guidelines for clinical practice. Progrès en Urologie. 2016; 26(1):S61-S72. doi: https://doi. org/10.1016/S1166-7087(16)30429-8

11. Wheeler TL, Gerten KA, Garris JB. Obliterative vaginal surgery for pelvic organ prolapse. Obstet Gynecol Clin North Am. 2009; 36(3):637-58. doi: https://doi.org/10.1016/j.ogc.2009.08.003

12. FitzGerald MP, Richter HE, Siddique S, Thompson P, Zyczynski H; Ann Weber for the Pelvic Floor Disorders Network. Colpocleisis: a review. Int Urogynecol J Pelvic Floor Dysfunct. 2006; 17(3):261-71. doi: https://doi.org/10.1007/s00192-005-1339$\underline{9}$

13. Marín Ardila L. Colpocleisis de Le Fort. Rev Colomb Obstet Ginecol. 1966; 17(6):41524. doi: https://doi.org/10.18597/rcog.2647

14. Bump RC, Mattiasson A, Bo K, Brubaker LP, DeLancey JO, Klarskov P, Shull BL. The standardization of terminology of female pelvic organ prolapse and pelvic floor dysfunction. Am J Obstet Gynecol. 1996; 175(1):10-7. doi: https://doi.org/10.1016/ S0002-9378(96)70243-0

15. Yalcin I, Bump RC. Validation of two global impression questionnaires for incontinence. Am J Obstet Gynecol. 2003; 189(1): 98- 101.doi: https://doi.org/10.1067/ mob.2003.379

16. Srikrishna S, Robinson D, Cardozo L. Validation of the Patient Global Impression of Improvement (PGI-I) for urogenital prolapse. Int Urogynecol J. 2010; 21(5): 523- 8. doi: https://doi.org/10.1007/s00192-0091069-5

17. Handa VL, Garrett E, Hendrix S, Gold E, Robbins J. Progression and remission of pelvic organ prolapse: a longitudinal study of menopausal women. Am J Obstet Gyne- col. 2004; 190(1):27-32. doi: https://doi.org/10.1016/j.ajog.2003.07.017

18. Hernández T MC, Gómez L M, Echavarria R LG. Colpocleisis sitio-específica: 3 años de experiencia. Rev. chil. obstet. ginecol. 2018; 83(3): 219-27. doi: http://dx.doi. org/10.4067/s0717-75262018000300219

19. Valentina De Petris V, Domingo Laiz R, Francisco Castro L. Técnicas obliterativas en el tratamiento quirúrgico del prolapso genital femenino. Experiencia en el Servicio de Salud Metropolitano Oriente, Santiago-Chile. Rev. chil. obstet. ginecol. 2017; 82(5):480-9. doi: http://dx.doi.org/10.4067/ s0717-75262017000500480

20. Conde P, Grilo i, Martins A, Martins P, Moniz A. Cirurgia obliterativa do prolapso dos órgãos pélvicos - uma opção ainda válida? Acta obstet Ginecol Port 2010; 4(3):12631. Recuperado a partir de: http://hdl.handle.net/10400.17/1333

21. Espitia-De La Hoz. Evaluación de la calidad de vida en mujeres climatéricas con prolapso genital luego del uso del pesario. Rev. Col. de Menopausia. 2018; 24(4):7-18.doi: http://asomenopausia.com/r/24_4.pdf\#page $=8$

22. Espitia-De La Hoz, Orozco-Santiago L, Orozco-Gallego H. Eficacia del reparo de la procidencia genital completa en la recuperación de la sexualidad de las mujeres intervenidas quirúrgicamente. Rev. Col. de Menopausia. 2018; 24 (1):21-31. Recuperado a partir de: http://asomenopausia. com/r/24_1.pdf

23. Zebede S, Smith AL, Plowright LN, Hegde A, Aguilar VC, Davila GW. Obliterative LeFort colpocleisis in a large group of elderly women. Obstet Gynecol. 2013; 121(2 Pt 1):279-84. doi: 10.1097/AOG.0b013e$31827 \mathrm{~d} 8 \mathrm{fdb}$

24. Hatch Q, Steele SR. Rectal prolapse and intussusception. Gastroenterol Clin North Am. 2013; 42(4):837-61. doi: 10.1016/j. gtc. 2013.08.002

25. Mikos T, Chatzipanteli M, Grimbizis GF, Tarlatzis BC. Enlightening the mechanisms of POP recurrence after LeFort colpocleisis. Case report and review. Int Urogy- 
necol J. 2016; 28:971-8. doi: https://doi. org/10.1007/s00192-016-3236-9

26. Catanzarite T, Rambachan A, Mueller MG, Pilecki MA, Kim JY2, Kenton K. Risk factors for 30-day perioperative complications after Le Fort colpocleisis. J Urol. 2014; 192(3):788-92. doi: https://doi.org/10.1016/j.juro.2014.03.040

27. Hill AJ, Walters MD, Unger CA. Perioperative adverse events associated with colpocleisis for uterovaginal and posthysterectomy vaginal vault prolapse. Am J Obstet Gynecol 2016; 214:501.e1-6. doi: https:// doi.org/10.1016/j.ajog.2015.10.921

28. Krissi H, Aviram A, Eitan R, From A, Wiznitzer A, Peled Y. Risk factors for recurrence after Le Fort colpocleisis for severe pelvic organ prolapse in elderly women. International Journal of Surgery. 2015; 75-9. doi: https://doi.org/10.1016/j.ijsu.2015.06.026

29. Park JY, Han SJ, Kim JH, Chun KC, Lee TS. Le Fort partial colpocleisis as an effective treatment option for advanced apical prolapse in elderly women. Taiwan J Obstet Gynecol. 2019; 58(2):206-11. doi: https:// doi.org/10.1016/j.tjog.2019.01.007

30. Espitia-De La Hoz FJ. Manejo quirúrgico del prolapso de la cúpula vaginal utilizando mallas de polipropileno y el abordaje por vía vaginal con la fijación bilateral al ligamento sacroespinoso. Rev Colomb de Menopausia. 2017; 23(2):70-8. Recuperado a partir de: http://asomenopausia. com $/ r / 23$ 2.pdf

31. Jones KA, Zhuo Y, Solak S, Harmanli O. Hysterectomy at the time of colpocleisis: a decision analysis. Int Urogynecol J. 2016; 27(5):805-10. doi: https://doi.org/10.1007/ s00192-015-2903-6

32. Glavind K, Kempf L. Colpectomy or Le Fort colpocleisis a good option in selected elderly patients. International Urogynecology Journal, 2004; 16(1):48-51. doi: https://doi. org/10.1007/s00192-004-1218-9

33. Espitia-De La Hoz FJ. Menopause and sexuality: characterization of sexual dysfunctions during climacteric, in women of Quindío (Colombia). Obstet Gynecol Int J. 2019; 10(6):419-24. doi: 10.15406/ ogij.2019.10.00477 